\title{
Modbus HMI bluetooth for outseal PLC
}

Cite as: AIP Conference Proceedings 2217, 030124 (2020); https://doi.org/10.1063/5.0000583

Published Online: 14 April 2020

\section{Djoko Untoro Suwarno}

\section{Lock-in Amplifiers up to $600 \mathrm{MHz}$

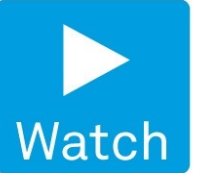

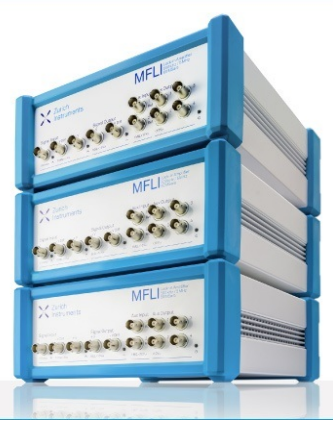




\title{
Modbus HMI Bluetooth for Outseal PLC
}

\author{
Djoko Untoro Suwarno
}

\begin{abstract}
Electrical Engineering Department, Faculty of Science and Technology, Sanata Dharma University, Yogyakarta, Indonesia
\end{abstract}

Corresponding author: joko_unt@usd.ac.id

\begin{abstract}
The PLC is widely used as a controller with input in the form of switches and sensors and output in the form of coils. PLC can be connected with HMI to facilitate the operation of the PLC. The usual HMI is installed on a PLC in the form of a stand-alone PC HMI and HMI that is connected via serial cable or Ethernet cable. The HMI in the form of wireless is rarely found. In this paper, will show the use of HMI connected with Bluetooth (BT) on the Outseal PLC (locally made PLC). The PLC is programmed with standard ladder diagram. Selector 4 channels with input in the form of switches and output in the form of coils (lights). Lights can be selected via the switch. Difficulties in the PLC with input using the switch are not easily changed from afar. HMI designed has facilities available for ALL OFF, ALL ON and TOGGLE mode. The HMI uses a smart phone with HMI modbus apk for android. The Smart phone screen can be used as a monitor and is a touch screen for input. Screen size of 5.5 inch, pixel size of 7201280 is capable of displaying 7 switches and 4 indicators
\end{abstract}

\section{INTRODUCTION}

The PLC is widely used for controllers in industry. The simplest PLC unit is a CPU with an input in the form of a switch and an output in the form of a coil. To display and control the PLC used HMI (Human Machine Interface) which is in the form of a touch screen. On the HMI screen can be filled with input and display devices. The widely available HMIs are PC monitor screens as HMIs and dedicated HMIs. HMI communication can be done using serial communication via an attached cable

Problem to solve in this paper: Selector 4 channels of relays starting all the OFF condition. The channel can be controlled ON or OFF directly using the switch. There is an option to change the Indi dually channel into ON of OFF condition. The other option is to change all condition into ON condition. The next option is all OFF and the reverse choice from the original state using remote access.

\section{THE THEORETICAL BACKGROUND}

The Outseal PLC is an Arduino-based PLC that has been loaded with a special boat loader [1]. The PLC input part that is connected to the outside world is $\mathrm{S} 1$ to $\mathrm{S} 8$, while the output part is connected via the relay coil R1 to R8. There is a memory bit with names B1 through B128. Input ports and output ports can be expanded up to 128 ports

Modbus protocol is a protocol for communication on PLC with other equipment such as HMI [2]. Modbus communication between master and slave can be via serial cable (modbus RTU) or TCP / IP (modbus TCP / IP).

The 5th International Conference on Industrial, Mechanical, Electrical, and Chemical Engineering 2019 (ICIMECE 2019)

AIP Conf. Proc. 2217, 030124-1-030124-6; https://doi.org/10.1063/5.0000583

Published by AIP Publishing. 978-0-7354-1971-1/\$30.00 
Modbus protocol, the master gives the command (function) and the address to the slave. The data format on the RTU modbus is shown in the Figure 1.

Kuang [3] conducted a communication experiment between Arduino and PLC using the Modbus protocol. PLC as modbus master and Arduino as slave modbus

\begin{tabular}{|c|c|c|c|c|c|}
\hline & \multicolumn{4}{|c|}{ MODBUS message } & \\
\hline Start & Address & Function & Data & CRC Check & End \\
\hline$\geq 3.5$ char & 8 bits & 8 bits & $\mathrm{N} \times 8$ bits & 16 bits & $\geq 3.5$ char \\
\hline
\end{tabular}

FIGURE 1.The data format for modbus RTU

Modbus function and addresses are presented in the TABLE 1 and TABLE 2

TABLE 1 Modbus functions code

\begin{tabular}{cl}
\hline Modbus function code & Description \\
\hline 0x01 & Read Coil / Bit Status \\
0x02 & Read Switch Status \\
0x03 & Read Holding Register \\
0x04 & Read Internal Register \\
0x05 & Write Single Coil \\
0x06 & Write Single Register \\
0x0F & Write multiple Register \\
\hline
\end{tabular}

TABLE 2 Modbus address

\begin{tabular}{lllrr}
\hline Table Name PLC & \multirow{2}{*}{ object } & permission & \multicolumn{1}{l}{ Address } & \multicolumn{1}{c}{ Address offset } \\
\hline R1... R128 & Coil Relay & Read only & $0 \ldots 9999$ & $0-127$ \\
B1... B128 & Bit & Read / Write & $0 \ldots 9999$ & $128-255$ \\
S1... S128 & Switch & Read only & $10000-19999$ & $0-127$ \\
I.1.. I.40 & Internal Register & Read / write & $40000-49999$ & $1 . .100$ \\
H1... H500 & Holding Register & Read// Write & $40000-49999$ & $500-1000$ \\
\hline
\end{tabular}

Ladder diagram shows the logic used. The logic used is IDENTITY logic, NOT logic, AND logic, OR and EXOR logic. Logical operations according to Boole are shown in the TABLE 3.

TABLE 3.The Boolean algebra for logic operation [4]

\begin{tabular}{ccc}
\hline Logic AND operation & Logic OR operation & Logic XOR operation \\
\hline$A 0=0$ & $A+0=A$ & $A \otimes 0=A$ \\
$A \cdot 1=A$ & $A+1=1$ & $A \otimes 1=\bar{A}$ \\
$A \cdot A=A$ & $A+A=A$ & $A \otimes A=1$ \\
$A \cdot \bar{A}=0$ & $A+\bar{A}=1$ & $A \otimes \bar{A}=0$ \\
\hline
\end{tabular}

In the logic operation table according to Boole, the logic operation AND produces two conditions namely A, 0 . The output is 0 (FALSE) if one of the inputs is 0 (FALSE). In OR logic operations, outputs are valued at A, 1. Output is 1 (TRUE) when one input is 1 (TRUE). In XOR operations output can be A, NOT A, 1, 0. Output is A when input is 0 while output is NOT A when input is 1. 


\section{METHODOLOGY}

The equipment used in this research are the Outseal Studio Release 10 rev 1 from www.outseal.com, an Outseal PLC board V3, a Bluetooth module and a Smartphone with HMI modbus apk (available on play store) as shown in Figure 2 2. The PLC outseal board is programmed in ladder diagram using the Outseal studio. Interaction between PLC and humans using HMI which is designed on a smartphone. The next problem is designing and implementing HMI on smart phones

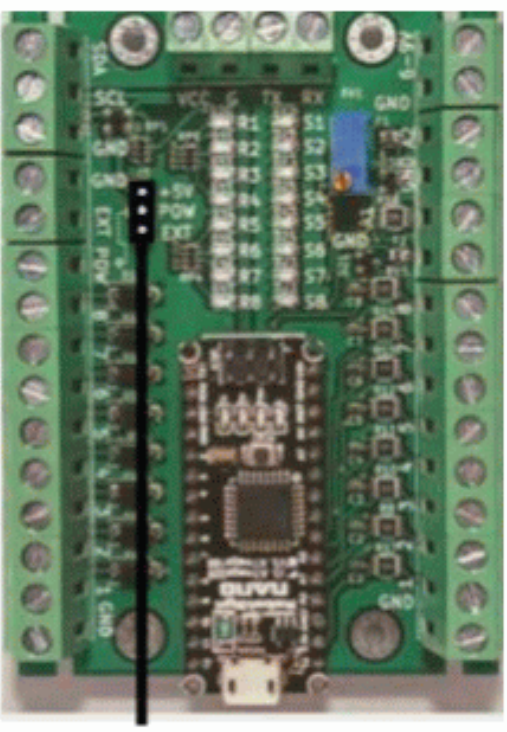

The PLC outseal + modbus RTU (source http://www.outseal.com)

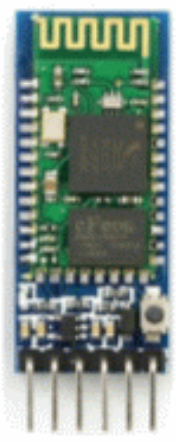

\section{Bluetooth $\mathrm{HCO5}$}

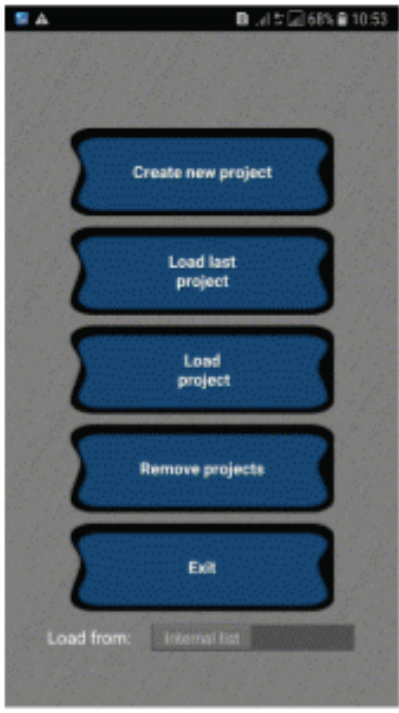

\section{Smartphone + Bluetooth}

+ HMI modbus apk

FIGURE 2. Setup experiment the PLC outseal + HMI Bluettoh

Diagram ladder for Selector 4 channels controlled by HMI android Bluetooth shown in Figure 3 and Figure 4
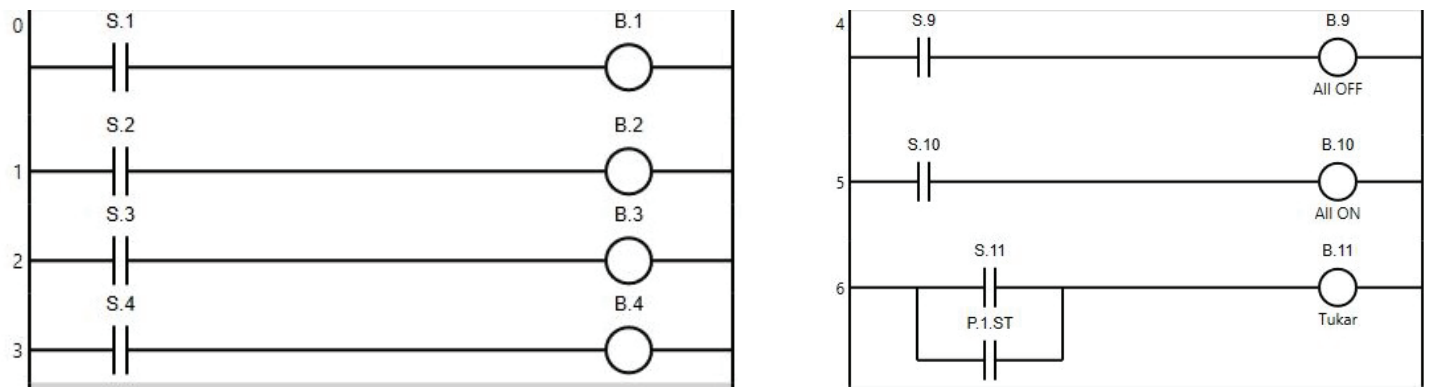

FIGURE 3. Ladder diagram for input from switch (rung 0 - 3), rung $4-6$ select mode ALL ON, ALL OFF, TOGGLE
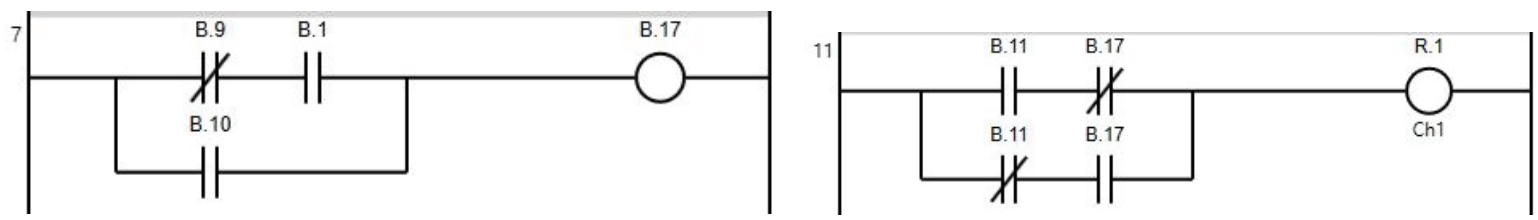

FIGURE 4..(a) Ladder diagram of ALL ON mode and ALL OFF mode. (b) ladder diagram for TOGGLE mode 
In rung 0 to rung $3, \mathrm{~S} 1$ to $\mathrm{S} 4$ are connected with an external switch to activate channels 1 to 4 . S1 cannot be connected directly to R1 but is connected to B1 (memory bit). In rung 7 and rung 11 shows the logic implementation for ALL OFF, ALL ON and TOGGLE modes.

Address table and Request type on the HMI created are presented in the TABLE 4.

TABLE 4. Setting object in HMI modbus

\begin{tabular}{|c|c|c|c|c|c|}
\hline Object & Label & Ladder PLC & Modbus Address & Request Type & Function Code \\
\hline & $\mathrm{CH} 1$ & I. 1 & 0 & Int $16,0=\mathrm{OFF}, 1=\mathrm{ON}$ & $\begin{array}{c}\text { Read Holding } \\
\text { Reg } \\
\text { 0x03 }\end{array}$ \\
\hline & $\mathrm{CH} 2$ & I. 2 & 1 & Int $16,0=\mathrm{OFF}, 1=\mathrm{ON}$ & 0x03 \\
\hline & $\mathrm{CH} 3$ & I. 3 & 2 & Int $16,0=\mathrm{OFF}, 1=\mathrm{ON}$ & 0x03 \\
\hline & $\mathrm{CH} 4$ & I. 4 & 3 & Int $16,0=\mathrm{OFF}, 1=\mathrm{ON}$ & 0x03 \\
\hline & ALL OFF & I.9 & 8 & Int $16,0=\mathrm{OFF}, 1=\mathrm{ON}$ & 0x03 \\
\hline & ALL ON & I. 10 & 9 & Int $16,0=\mathrm{OFF}, 1=\mathrm{ON}$ & 0x03 \\
\hline & TOGGLE & I.11 & 10 & Int $16,0=\mathrm{OFF}, 1=\mathrm{ON}$ & $0 \times 03$ \\
\hline & & R1 & 0 & $\begin{array}{l}\text { Read Bit State } \\
\text { (switch) }\end{array}$ & $\begin{array}{c}\text { Read coil status } \\
\text { 0x01 }\end{array}$ \\
\hline & & $\mathrm{R} 2$ & 1 & $\begin{array}{l}\text { Read Bit State } \\
\quad \text { (switch) }\end{array}$ & 0x01 \\
\hline & & R3 & 2 & $\begin{array}{l}\text { Read Bit State } \\
\text { (switch) }\end{array}$ & 0x01 \\
\hline & & R4 & 3 & $\begin{array}{l}\text { Read Bit State } \\
\text { (switch) }\end{array}$ & 0x01 \\
\hline & & R8 & 7 & $\begin{array}{l}\text { Read Bit State } \\
\quad \text { (switch) }\end{array}$ & 0x01 \\
\hline
\end{tabular}

\section{RESULTS AND DISCUSSION}

HMI results made are presented in the following figure. The HMI consists of only one screen and contains $4 \mathrm{Ch} 1$, $\mathrm{Ch} 2 \mathrm{Ch} 3$ and $\mathrm{Ch} 4$ buttons to select channels, 3 buttons to select modes: ALL OFF, ALL ON and TOGGLE. There are 4 outputs in the form of lights and 1 indicator to show the HMI is connected to the PLC.There are 4 views to test each mode. In direct mode the output state matches the switch state. In ALL OFF mode all lights go out even though the input value is correct. In ALL ON mode all output states are TRUE even though the input is FALSE. In TOGGLE mode the output state is the opposite of the input state. When the input is true, the output is false, when the input is false, the output is true. The complete logic operation for Selector 4 channel show in TABLE 5. 
TABLE 5 Logic Operation used in Selector 4 channels

\begin{tabular}{ll}
\hline Operation & Description \\
\hline $\mathrm{S} 1 \rightarrow \mathrm{B} 1, \mathrm{~S} 2 \rightarrow \mathrm{B} 2, \mathrm{~S} 3 \rightarrow \mathrm{B} 3, \mathrm{~S} 4 \rightarrow \mathrm{B} 4$ & Input from the Switch \\
$\mathrm{I} .1 \rightarrow \mathrm{B} 9$ & I.1 is activated from the HMI as the ALL OFF button \\
$\mathrm{I} .2 \rightarrow \mathrm{B} 10$ & I.2 is activated from the HMI as the ALL ON button \\
L3 $\rightarrow$ B11 & I.3 is activated from the HMI as the TOGGLE button \\
$((\mathrm{B} 1$ AND B9) OR B10) XOR B11 $\rightarrow$ R1 & Output to Coil Relay R1 \\
$((\mathrm{B} 2$ AND B9) OR B10) XOR B11 $\rightarrow$ R2 & Output to Coil Relay R2 \\
$((\mathrm{B} 3$ AND B9) OR B10) XOR B11 $\rightarrow$ R3 & Output to Coil Relay R3 \\
$((\mathrm{B} 4$ AND B9) OR B10) XOR B11 $\rightarrow$ R4 & Output to Coil Relay R4 \\
\hline
\end{tabular}

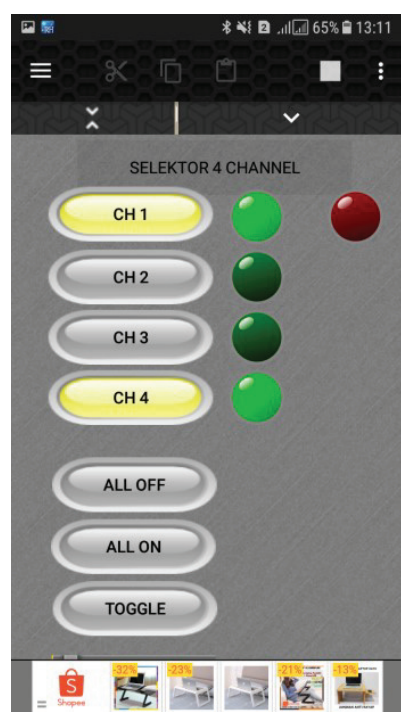

(a)

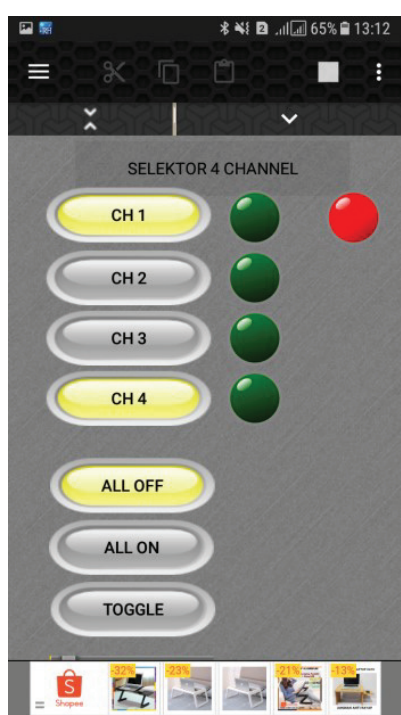

(b)

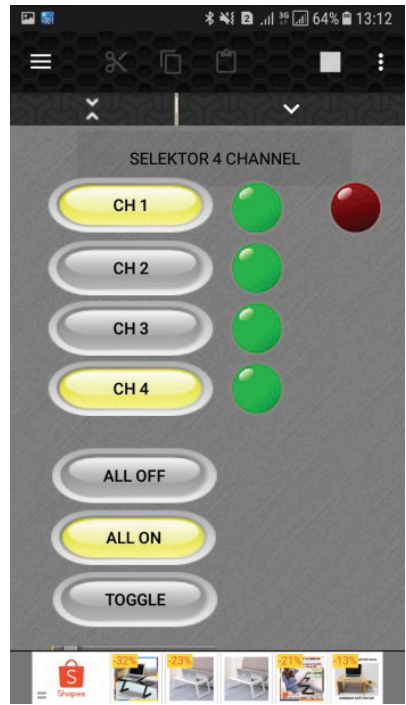

(c)

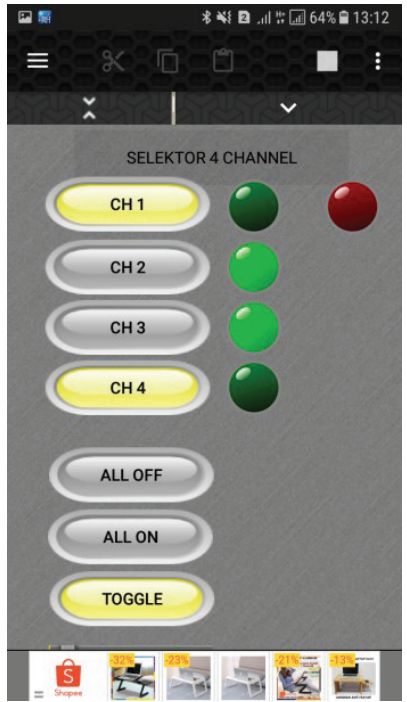

(d)

FIGURE 5 (a) Selector for 4 channels with Direct Mode. Push button with CH1 label will activate Green Lamp \#1. (b), in ALL OFF mode, all output become OFF condition whatever input conditions. (c) in ALL ON mode, all output become ON condition whatever input conditions. (d) in TOGGLE mode, the outputs will negation from input

Button ALL ON, ALL OFF and button TOGGLE operate individually, not allow activating together. The comparison between HMI created with HMI design Tips shown in table 6

TABLE 6. Comparison HMI created with McDaniel [5] HMI design tips

\begin{tabular}{lcl}
\hline HMI design Tips & HMI created & Comments \\
\hline Create a storyboard & Not apply & Just one page \\
Talk to operators & Yes & Operated by other user \\
Use colour and animation judiciously & Yes & Colour default from apk \\
Add graphics & No & Don't need graphics \\
Display appropriate images & Yes & Image of switches and lamps \\
Keep important items available & Yes & All item has a function \\
Offer situational awareness & No & \\
Limit required screen access clicks & Yes & User need to open main HMI page and select \\
& & HMI project \\
Give feedback and lead the way & Not yet & \\
Beware of pop-ups & ok & There are promotion pop up plug in (free \\
& & android apk) \\
Use date and time stamp logging & Not yet & No logging function \\
Use appropriate password protection & no & Single user, password from smartphone \\
Create a style guide & yes & Using template from apk \\
\hline
\end{tabular}


HMI design using HMI modbus for android is free apk, but its apk with pop up advertising. To remove pop up advertising user must pay for registration to apk creator.

In making HMI, it is necessary to pay attention to the esthetics and functionality of the object used. It should also be noted that the parameters used for each object are the slave PLC address used, modbus address, request type.

Benefit using android smartphone as HMI is cheap and easy to use. Now Bluetooth communication is standard in smartphone and easy to access. Bluetooth baud rate used in this project is $57600 \mathrm{bps}$.

\section{CONCLUSIONS}

1. Communication between the Outseal PLC and HMI android using Bluetooth with baud rate 57600 bps

2. Communication Protocol used in this project is Modbus RTU

3. HMI created success with all function button work done, and fit from HMI design tips.

4. Using smartphone android as HMI is cheap and easy to build.

\section{ACKNOWLEDGEMENT}

The author would like to thank the outseal for the support of the outbound PLC, especially to Mr. Agung Bakthiar and Mr. Dian Eko Prasetyo, and Mr. Petrus Setyo Prabowo for the financial support of this conference.

\section{REFERENCES}

1. Bakhtiar, A. (2019). Panduan Dasar Outseal PLC (in Indonesian). www.outseal.com.

2. www.modbus.org. (2002). MODBUS over serial line specification and implementation guide V1.0. modbus.org.

3. Yu-cong Kuang, (2012), Communication Between PLC and Arduino Based on Modbus Protocol, 2014 Fourth International Conference on Instrumentation and Measurement, Computer, Communication and Control

4. Givant, S. R., \& Halmos, P. R. (2009). Introduction to Boolean algebras. Springer.

5. McDaniel, C. (2015). HMI design, Baker's dozen tips to create more effective HMIs. ISA publications, InTech Magazine, Automation IT. 